\title{
Bessel inequality for robust stability analysis of time-delay system
}

\author{
F. Gouaisbaut ${ }^{a, b}$, Y. Ariba ${ }^{a, c}$ and A. Seuret ${ }^{a, d}$
}

\begin{abstract}
This paper addresses the problem of the stability analysis for a linear time-delay systems via a robust analysis approach and especially the quadratic separation framework. To this end, we use the Bessel inequality for building operators that depend on the delay. They not only allow us to model the system as an uncertain feedback system but also to control the accuracy of the approximations made. Then, a set of LMIs conditions are proposed which tends on examples to the analytical bounds for both delay dependent stability and delay range stability.
\end{abstract}

\section{INTRODUCTION}

The rapid development of networked controlled systems has highlighted the need to understand why and how a transmission delay could destabilize an overall closed-loop system. For over a decade, many researchers have thus studied stability of delay systems and nowadays several methodologies are well established. Among the most interesting are the analytical methods based on the study of the characteristic equation [1], [2], which often lead to non conservative results. The other side of the coin is that such methods are not suitable for uncertain systems and timevarying delay systems. Other popular techniques are those based on the search of a Lyapunov functional ([3] and references therein). Indeed, if a linear delay system is stable, a Lyapunov functional, called complete Lyapunov functional, may be built. Nevertheless, it reveals itself quite complicated because these parameters are solutions of a partial differential equation [4]. Many works are therefore dedicated to the construction of approximate complete Lyapunov functionals by considering extended state variables [5], [6] at the cost of an increased computational complexity. At the heart of all these techniques are the use of integral inequalities as Jensen's [7] or Wirtinger's ones [8], [9], [10]. These inequalities are essential and have been developed to take advantage of information on delayed signals and to reduce the conservatism induced when one bounds the derivative of the Lyapunov functional. The obtained criteria are then often transformed into LMIs criteria through the use of slack variables [11], [12], [13], [14]. Note that all these techniques have been extended to various cases like time varying delay or uncertain systems. At last, some methods lie on the use of robust analysis methods such as Scaled Small Gain Theorem

\footnotetext{
${ }^{a}$ CNRS, LAAS, 7 avenue du Colonel Roche, 31077 Toulouse, France.

${ }^{b}$ Univ de Toulouse, UPS, LAAS, F-31400, Toulouse, France.

${ }^{c}$ Dpt of Electrical Engineering and Computer Science, ICAM, 75 avenue de Grande Bretagne, 31300 Toulouse, France.

${ }^{d}$ Univ de Toulouse, LAAS, F-31400 Toulouse, France.

\{fgouaisb, aseuret, yariba\}@laas.fr

This work has been funded by the ANR under grant LIMICOS ANR-12BS03-0005
}

[15], [16], IQCs [17] or Quadratic Separation [18]. In this context, the general idea is to consider the delay system as an uncertain linear finite dimensional system. More precisely, the trick is to model the delay system as a nominal finite dimensional system in closed loop with some uncertainties whose characteristics depend on the uncertain delay. Once the modeling completed, a final work is to characterize the uncertainty as precisely as possible. Generally, this uncertainty is highly structured and is not a rational function with respect to $s$. This difficulty is overcome by covering the delay uncertainty by a class of larger unstructured uncertainties whose bounds depend directly on the value of the delay [16]. This last step brings a lot of conservatism and many studies have been conducted to understand the ins and outs [3].

In this paper, we are interested in studying stability of a linear time delay system by use of the quadratic separation concept, known to give interesting results [19]. The interconnected model is provided by introducing a set of uncertainties which can be viewed as the projection of a delay operator to the set of Legendre polynomials. Once the model is designed, the uncertainty set is embedded into a more suitable uncertainty by the use of Bessel inequality. Invoking classical quadratic separation theorem, it results a series of LMIs conditions less and less conservative as the sequence of Legendre polynomials is increasing. Note that two different types of results are proposed, a pointwise delay stability theorem and a delay-range stability theorem. On numerical examples, our results show a clear improvement for the delay bound and a reduction of numerical complexity. The paper is organized as follows. Section 2 is devoted to the preliminaries and especially recalls Bessel inequality. Section 3 provides with a first result based on Bessel inequality. Then, section 4 develop the main results, a set of less and less conservative LMIs conditions for the stability analysis. Two types of results are proposed, a pointwise theorem and a delay range theorem. Section 5 is dedicated to the illustration of our techniques on some examples extracted from the literature. Notations: Throughout the paper, the following notations are used. $1_{\mathrm{n}}$ and $0_{\mathrm{m} \times \mathrm{n}}$ denote respectively the identity matrix of size $n$ and null matrix of size $m \times n$. If the context allows it, the dimensions of these matrices will be omitted. For two symmetric matrices, $A$ and $B, A>(\geq) B$ means that $A-B$ is (semi-) positive definite. $A^{T}$ denotes the transpose of $A$. $\operatorname{diag}(A, B, C)$ stands for the block diagonal matrix.

\section{PRELIMINARIES}

\section{A. Definitions and problem statement}

We define by $H$ the vector space of complex valued square integrable functions on $[-h, 0]$. For any functions in $H$, we 
define the hermitian inner product

$$
\langle f, g\rangle=\int_{-h}^{0} f(\theta) g^{*}(\theta) d \theta,
$$

where $f$ and $g$ belong to $H$. Basically, $x_{i}, x_{j} \in H, i \neq j$ are orthogonal if and only if $\left\langle x_{i}, x_{j}\right\rangle=0$. A sequence of $H,\left\{e_{0}, \ldots, e_{n}\right\}$ is an orthonormal sequence if and only if $\left\langle e_{i}, e_{j}\right\rangle=\kappa_{i j}$, where $\kappa_{i j}$ is the kronecker notation. Based on these definitions, we recall the Bessel inequality that will be employed later in the paper.

Lemma 1 (Bessel inequality): Let $\left\{e_{0}, e_{1}, e_{2}, \ldots, e_{n}\right\}$ be an orthonormal sequence in $H$. Then, for any $f$ in $H$ the following inequality holds:

$$
\langle f, f\rangle \geq \sum_{i=0}^{n}\left|\left\langle f, e_{i}\right\rangle\right|^{2}
$$

\section{B. Problem statement}

We consider the following time-delay system:

$$
\dot{x}(t)=A x(t)+A_{d} x(t-h)
$$

where $x(t) \in \mathbb{R}^{n}$ is the state vector, $A$ and $A_{d} \in \mathbb{R}^{n \times n}$ are constant matrices. The constant $h$ is an unknown delay belonging to an interval $\left[h_{\min }, h_{\max }\right]$. We aim at proving stability of the time delay system (1) for all delays belonging to this interval. To this end, we propose to employ the concept of robust analysis widely used in the literature and more specifically the principle of quadratic separation [19]. The key idea is to reformulate the delay system (1) as an uncertain feedback system represented by the Figure 1. The uncertainty $\nabla$ is constructed according to the delay dynamics [18], [17]. Then, the stability conditions follow by invoking the theorem introduced below.

Let consider the interconnection defined by Figure 1 where

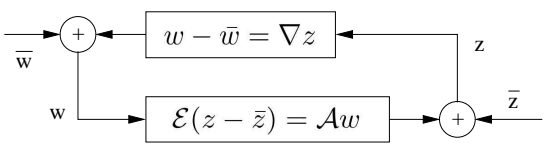

Fig. 1. Feedback system.

$\mathcal{E}$ and $\mathcal{A}$ are two, real valued, possibly non-square matrices and $\nabla$ is a linear operator which represents the system uncertainties. This latter is assumed to belong to an uncertain set $\mathbb{W}$. For simplicity, we assume that $\mathcal{E}$ is full column rank.

Theorem 1 ([18]): The uncertain feedback system of Figure 1 is well-posed and stable if and only if there exists a Hermitian matrix $\Theta=\Theta^{*}$ satisfying both conditions

$$
\begin{aligned}
& {\left[\begin{array}{cc}
\mathcal{E} & -\mathcal{A}
\end{array}\right]^{\perp *} \Theta\left[\begin{array}{ll}
\mathcal{E} & -\mathcal{A}
\end{array}\right]^{\perp}>0,} \\
& {\left[\begin{array}{c}
1 \\
\nabla
\end{array}\right]^{*} \Theta\left[\begin{array}{c}
1 \\
\nabla
\end{array}\right] \leq 0 \quad, \quad \forall \nabla \in \mathbb{W} .}
\end{aligned}
$$

\section{AN INSTRUCTIVE RESULT}

\section{A. Some interesting inequalities}

We aim at finding out some operators to describe accurately the dynamics of the delay system. These operators are then embedded into normed bounded uncertainties much more easy to handle in a robust analysis concept. First of all, let define a delay function $f$ expressed using Laplace transform;

$$
f:\left\{\begin{array}{cl}
{[-h, 0]} & \rightarrow \mathbb{C} \\
\theta & \mapsto f(\theta)=e^{s \theta}
\end{array}\right.
$$

where $s \in \mathbb{C}^{+}$. Obviously, $f \in H$ and we get the following lemma:

Lemma 2: Considering the delay function $f$, defined in (4), the inequality $\langle f, f\rangle \leq h$ holds.

Proof: omitted.

Consider now the two polynomials defined on $\theta \in[-h, 0]$ by:

$$
e_{0}(\theta)=\frac{1}{\sqrt{h}}, e_{1}(\theta)=\sqrt{\frac{3}{h}}\left(\frac{2}{h} \theta+1\right) .
$$

It is straightforward to verify that these polynomials belong to $H$ and satisfy the conditions $\left\langle e_{0}, e_{0}\right\rangle=\left\langle e_{1}, e_{1}\right\rangle=$ 1 and $\left\langle e_{0}, e_{1}\right\rangle=0$. Hence, the pair $\left\{e_{0}, e_{1}\right\}$ forms an orthonormal sequence and applying Lemma 1, we have:

$$
\langle f, f\rangle \geq\left|\left\langle f, e_{0}\right\rangle\right|^{2}+\left|\left\langle f, e_{1}\right\rangle\right|^{2} .
$$

This last inequality puts us on the right track to set up two delay-related operators $\delta_{0}$ and $\delta_{1}$ :

$$
\begin{gathered}
\delta_{0}=\sqrt{h}\left\langle f, e_{0}\right\rangle=\int_{-h}^{0} e^{s \theta} d \theta \\
\delta_{1}=\sqrt{\frac{h}{3}}\left\langle f, e_{1}\right\rangle=\int_{-h}^{0} e^{s \theta}\left(\frac{2}{h} \theta+1\right) d \theta,
\end{gathered}
$$

Using the result of Lemma 2, it is proved that

$$
\delta_{0} \delta_{0}^{*}+3 \delta_{1} \delta_{1}^{*} \leq h^{2} .
$$

It allows us to embed the operators together into a disk of radius $h$.

Remark 1 (Jensen'inequality): Obviously, $\left\{e_{0}\right\}$ forms also an orthogonal sequence and the inequality $\delta_{0} \delta_{0}^{*} \leq h^{2}$ is still true. This one, closely related to Jensen inequality has been widely used to produce sufficient stability conditions [20], [16]. Adding a second uncertainty $\delta_{1}$ can clearly reduce the conservatism associated with the Jensen inequality at the cost of an increasing complexity for $\nabla$.

\section{B. Modeling the delay system as an uncertain system}

The main problem consists in modeling the delay system (1) into an interconnected system as depicted in Figure 1. The question is how can we describe (1) as precisely as possible and keeping a constructive and tractable condition. In this paper, the delay system is modeled with a set of uncertain blocks (e.g. $s^{-1}, \delta_{0}$ and $\delta_{1}$ ), which are then embedded in the uncertain block $\nabla$. Let choose the following set of operators (viewed as an uncertain block): 


$$
\nabla=\operatorname{diag}\left(s^{-1} 1_{2 \mathrm{n}}, e^{-h s} 1_{\mathrm{n}},\left[\begin{array}{l}
\delta_{0} 1_{\mathrm{n}} \\
\delta_{1} 1_{\mathrm{n}}
\end{array}\right]\right),
$$

which connects with the equation $w(t)=\nabla z(t)$,

$$
w(t)=\left[\begin{array}{c}
x(t) \\
\int_{t-h}^{t} x(\theta) d \theta \\
x(t-h) \\
\alpha(t) \\
\delta_{1}[\dot{x}(t)]
\end{array}\right] \text { and } z(t)=\left[\begin{array}{c}
\dot{x}(t) \\
\alpha(t) \\
x(t) \\
\dot{x}(t)
\end{array}\right],
$$

where $\alpha(t)=x(t)-x(t-h) . \delta_{0}$ and $\delta_{1}$ are defined in (6) and (7). The delay system (1) is then described by the equation

$$
\underbrace{\left[\begin{array}{llll}
1 & 0 & 0 & 0 \\
0 & 1 & 0 & 0 \\
0 & 0 & 1 & 0 \\
0 & 0 & 0 & 1 \\
0 & 0 & 0 & 0 \\
0 & 0 & 0 & 0
\end{array}\right]}_{\mathcal{E}} z(t)=\underbrace{\left[\begin{array}{ccccc}
A & 0 & A_{d} & 0 & 0 \\
1 & 0 & -1 & 0 & 0 \\
1 & 0 & 0 & 0 & 0 \\
A & 0 & A_{d} & 0 & 0 \\
1 & 0 & -1 & -1 & 0 \\
-1 & 2 / h & -1 & 0 & 1
\end{array}\right]}_{\mathcal{A}} w(t) .
$$

\section{A first stability condition}

Having proposed a model for the delay system, the uncertain set $\nabla$ is characterized as follows.

Lemma 3: A quadratic constraint for the operator $s^{-1}$ is given by the following inequality for any positive definite matrix $P$,

$$
\left[\begin{array}{c}
1_{\mathrm{n}} \\
s^{-1} 1_{\mathrm{n}}
\end{array}\right]^{*}\left[\begin{array}{cc}
0 & -P \\
-P & 0
\end{array}\right]\left[\begin{array}{c}
1_{\mathrm{n}} \\
s^{-1} 1_{\mathrm{n}}
\end{array}\right] \leq 0
$$

A quadratic constraint for the operator $e^{-h s}$ is given by the following inequality for any positive definite matrix $Q$,

$$
\left[\begin{array}{c}
1_{\mathrm{n}} \\
e^{-h s} 1_{\mathrm{n}}
\end{array}\right]^{*}\left[\begin{array}{cc}
-Q & 0 \\
0 & Q
\end{array}\right]\left[\begin{array}{c}
1_{\mathrm{n}} \\
e^{-h s} 1_{\mathrm{n}}
\end{array}\right] \leq 0
$$

The proof of this lemma can be found in [21], [22].

Lemma 4: A quadratic constraint for the operator $\left[\delta_{0}, \delta_{1}\right]^{T}$ is given by the following inequality for any positive definite matrix $R$,

$$
\left[\begin{array}{c}
1_{\mathrm{n}} \\
\delta_{0} 1_{\mathrm{n}} \\
\delta_{1} 1_{\mathrm{n}}
\end{array}\right]^{*}\left[\begin{array}{ccc}
-h^{2} R & 0 & 0 \\
0 & R & 0 \\
0 & 0 & 3 R
\end{array}\right]\left[\begin{array}{c}
1_{\mathrm{n}} \\
\delta_{0} 1_{\mathrm{n}} \\
\delta_{1} 1_{\mathrm{n}}
\end{array}\right] \leq 0 .
$$

Proof: Let consider $R^{1 / 2}$, the square-root of $R$, which is well defined since $R>0$. We apply Bessel inequality (8) to the signal $R^{1 / 2} f$ and it leads to

$$
\delta_{0} R \delta_{0}^{*}+3 \delta_{1} R \delta_{1}^{*}-h^{2} R \leq 0 .
$$

Factorizing on the left and on the right by $\left[1_{\mathrm{n}}, \delta_{0}^{*} 1_{\mathrm{n}}, \delta_{1}^{*} 1_{\mathrm{n}}\right]$ and its transposed conjugate, the result of the lemma is obtained.

Let now gather all the above lemmas to build the matrix inequality (3). For a matrix $\nabla$ defined in (9) and a separator of the form:

$$
\Theta=\left[\begin{array}{cc}
\Theta_{11} & \Theta_{12} \\
\Theta_{12}^{T} & \Theta_{22}
\end{array}\right]
$$

with $\Theta_{11}=\operatorname{diag}\left(0,-Q,-h^{2} R\right), \quad \Theta_{12}=$ $\left[\operatorname{diag}\left(-P, 0_{2 n}\right) \quad 0\right], \Theta_{22}=\operatorname{diag}(0, Q, R, 3 R)$, the constraint (3) holds.

A stability condition for time-delay systems of the form of (1) is then given in the following theorem.

Theorem 2: For a given positive scalar $h$, if there exist positive definite matrices $P \in \mathbb{R}^{2 n \times 2 n}, Q, R \in \mathbb{R}^{n \times n}$, then the system (1) is asymptotically stable if the following LMI is satisfied:

$$
\left[\begin{array}{ll}
\mathcal{E} & -\mathcal{A}
\end{array}\right]^{\perp *} \Theta\left[\begin{array}{ll}
\mathcal{E} & -\mathcal{A}
\end{array}\right]^{\perp}>0,
$$

where matrices $\mathcal{E}, \mathcal{A}$ and $\Theta$ are defined in (11) and (12).

Proof: It has been shown that system (1) could be expressed as in Figure 1 with (9)-(10)-(11). Using the separator $\Theta$ defined in (12) on the uncertainty $\nabla$ defined in (9), the condition (3) is satisfied. Hence, invoking Theorem 1 , the aforementioned system is stable if the first condition (2) is also satisfied with $\mathcal{E}, \mathcal{A}$ and $\Theta$ defined in (11) and (12).

\section{MAIN RESULTS}

This section is devoted to the extension of the previous theorem. The general idea is to build an increasing sequence of orthogonal polynomials. For a given $N \geq 1$, consider now a set of orthogonal polynomials $\left\{e_{0}, \ldots, e_{N}\right\}$ based on Legendre polynomials:

$$
e_{k}(\theta)=\sqrt{\frac{2 k+1}{h}} \cdot(-1)^{k} \sum_{l=0}^{k} p_{l}^{k}\left(\frac{\theta+h}{h}\right)^{l}, 0 \leq k \leq N,
$$

where $p_{l}^{k}=(-1)^{l}\left(\begin{array}{l}k \\ l\end{array}\right)\left(\begin{array}{l}k+l \\ l\end{array}\right)$. It can be proved that

$$
\left\{\begin{array}{l}
\left\langle e_{i}, e_{j}\right\rangle=0, \quad \text { for } i \neq j \\
\left\langle e_{i}, e_{i}\right\rangle=1 .
\end{array}\right.
$$

Following the procedure exposed in the last section, we define the corresponding operators $\delta_{k}, 0 \leq k \leq N$ :

$\delta_{k}=\sqrt{\frac{h}{2 k+1}}\left\langle f, e_{k}\right\rangle=\int_{-h}^{0}(-1)^{k} \sum_{l=0}^{k} p_{l}^{k}\left(\frac{\theta+h}{h}\right)^{l} e^{s \theta} d \theta$.

Consequently, the Bessel's inequality states that

$$
\sum_{k=0}^{N}(2 k+1) \delta_{k} \delta_{k}^{*} \leq h^{2} .
$$

At this stage, two different types of modeling are proposed. The first model will lead to a stability criterion for a pointwise delay, that is the criterion is only valid for a given delay $h$. By exploiting the special structure of the interconnection, the second model provides a delay range stability criterion, which ensures robust stability over an interval $\left[h_{\min }, h_{\max }\right]$.

\section{A. A pointwise delay stability condition}

Consider the following set of operators which can be viewed as an extension of the previous equation (9) for $\nabla$ :

$$
\nabla=\operatorname{diag}\left(s^{-1} 1_{\mathrm{n}(\mathrm{N}+1)}, e^{-h s} 1_{\mathrm{n}}, \tilde{\delta}_{N} \otimes 1_{\mathrm{n}}\right)
$$


where

$$
\tilde{\delta}_{k}=\left[\begin{array}{llll}
\delta_{0} & \delta_{1} & \vdots & \delta_{k}
\end{array}\right]^{T}
$$

It connects $w(t)$ and $z(t)$ via $w(t)=\nabla z(t)$ with

$$
w(t)=\left[\begin{array}{c}
x(t) \\
\tilde{\delta}_{N-1}[x(t)] \\
x(t-h) \\
\tilde{\delta}_{N}[\dot{x}(t)]
\end{array}\right] \text { and } z(t)=\left[\begin{array}{c}
\dot{x}(t) \\
\tilde{\delta}_{N-1}[\dot{x}(t)] \\
x(t) \\
\dot{x}(t)
\end{array}\right]
$$

It remains to model the delay system (1) using the signals $z(t)$ and $w(t)$ with a linear equation $\mathcal{E} z=\mathcal{A} w(t)$. This linear equation can be setup easily except the calculus of the term $\tilde{\delta}_{N}[\dot{x}(t)]$ which is tedious and have been placed in the Appendix VII. It shows that

$$
\begin{aligned}
\delta_{k}[\dot{x}]= & \int_{-h}^{0}(-1)^{k} \sum_{l=0}^{k} p_{l}^{k}\left(\frac{\theta+h}{h}\right)^{l} \dot{x}(t+\theta) d \theta, \\
= & x(t)-(-1)^{k} x(t-h) \\
& -\frac{1}{h}\left(U_{k} V^{-1} E \otimes 1_{n}\right) \tilde{\delta}_{N-1}[x(t)],
\end{aligned}
$$

where matrices $V$ and $U_{k}$ are respectively defined by equation (27) and (28) in the Appendix VII and $E=\left[\begin{array}{c}1_{N} \\ 0_{1 \times N}\end{array}\right]$. Finally, the feedforward linear equation can be written as $\mathcal{E} z=\mathcal{A} w(t)$ with

$$
\mathcal{E}=\left[\begin{array}{c}
1_{(N+3) n} \\
0_{(N+1) n \times(N+3) n}
\end{array}\right]
$$

and

$$
\mathcal{A}=\left[\begin{array}{c|ccc|c|cccc}
A & 0 & \ldots & 0 & A_{d} & 0 & \ldots & 0 & 0 \\
\hline 0 & 0 & \ldots & 0 & 0 & 1 & & 0 & 0 \\
\vdots & \vdots & & \vdots & \vdots & & \ddots & & \vdots \\
0 & 0 & \ldots & 0 & 0 & 0 & & 1 & 0 \\
\hline 1 & 0 & \ldots & 0 & 0 & 0 & \ldots & 0 & 0 \\
\hline A & 0 & \ldots & 0 & A_{d} & 0 & \ldots & 0 & 0 \\
\hline-1 & 0 & \ldots & 0 & 1 & 1 & 0 & \ldots & 0 \\
-1 & \frac{1}{h} W_{1} & -1 & 0 & 1 & & 0 \\
\vdots & & \vdots & \vdots & \vdots & & \ddots & \\
-1 & \frac{1}{h} W_{N} & (-1)^{N} 1 & 0 & 0 & & 1
\end{array}\right]
$$

with $W_{k}=U_{k} V^{-1} E \otimes 1_{n}$. We propose now the following pointwise delay stability theorem:

Theorem 3: For a given constant delay $h$, if there exist positive definite matrices $P \in \mathbb{R}^{(N+1) n \times(N+1) n}, Q, R \in$ $\mathbb{R}^{n \times n}$ then the system (1) is asymptotically stable for the given $h$ if the following LMI is satisfied:

$$
\left[\begin{array}{ll}
\mathcal{E} & -\mathcal{A}
\end{array}\right]^{\perp *} \Theta\left[\begin{array}{ll}
\mathcal{E} & -\mathcal{A}
\end{array}\right]^{\perp}>0
$$

where matrices $\mathcal{E}, \mathcal{A}$ and $\Theta$ are defined in (16) and (18).

Proof: We have shown that delay system (1) could be expressed as in Figure 1 with (14)-(15)-(16). Following the same procedure as in Theorem 2 and taking now into account the general Bessel inequality (13), a suitable separator is built:

$$
\Theta=\left[\begin{array}{ll}
\Theta_{1} & \Theta_{2} \\
\Theta_{2}^{*} & \Theta_{3}
\end{array}\right]
$$

with

$$
\begin{aligned}
& \Theta_{1}=\operatorname{diag}\left(0_{(N+1) n},-Q,-h^{2} R\right), \\
& \Theta_{2}=\operatorname{diag}\left(-P, 0_{n}, 0_{n \times(N+1) n}\right), \\
& \Theta_{3}=\operatorname{diag}\left(0_{(N+1) n}, Q, R, 3 R, \ldots,(2 N+1) R\right) .
\end{aligned}
$$

Thus, for $\nabla$ defined in (14) and the particular choice of $\Theta$ in (18), the condition (3) is satisfied. Finally, invoking Theorem 1, the aforementioned system is stable if the first condition (2) is also satisfied.

Remark 2: In this section, the case $N=0$ is not taken into account and has been already treated in [18]. Taking $N=1$ recovers Theorem 2 .

The proposed theorem proposes a stability result for a given $h$ and therefore may detect pockets of stability even if the system is unstable for the delay $h=0$. Furthermore, compared to the literature, the criterion has less variables to be optimized. Nevertheless, as it is exposed in subsection below, if the delay $h$ is uncertain, our criterion has to be slightly transformed in order to get a delay range stability criterion.

\section{B. A delay range delay stability condition}

The main idea is to consider that the delay is now an uncertain parameter belonging to the interval $\left[h_{\min }, h_{\max }\right]$. In that case, we propose the following interconnection which highlights this new uncertainty in the linear equation $w(t)=$ $\nabla z(t)$. The uncertain block is defined by

$$
\nabla=\operatorname{diag}\left(s^{-1} 1_{\mathrm{n}(\mathrm{N}+1)}, e^{-h s} 1_{\mathrm{n}}, \frac{1}{h} 1_{\mathrm{nN}}, \tilde{\delta}_{N} \otimes 1_{\mathrm{n}}\right),
$$

where $N \geq 1$. It connects the signals $w(t)$ and $z(t)$ defined by

$$
\left\{\begin{aligned}
w(t) & =\nabla z(t) \\
\mathcal{E} z(t) & =\mathcal{A} w(t)
\end{aligned}\right.
$$

with

$$
w(t)=\left[\begin{array}{c}
x(t) \\
\tilde{\delta}_{N-1}[x] \\
x(t-h) \\
y(t) \\
\tilde{\delta}_{N}[\dot{x}]
\end{array}\right], z(t)=\left[\begin{array}{c}
\dot{x}(t) \\
\tilde{\delta}_{N-1}[\dot{x}] \\
x(t) \\
\tilde{\delta}_{N-1}[x] \\
\dot{x}(t)
\end{array}\right]
$$

and $\mathcal{E}=\left[\begin{array}{c}1_{(2 N+3) n} \\ 0_{(N+1) n \times(2 N+3) n}\end{array}\right]$,

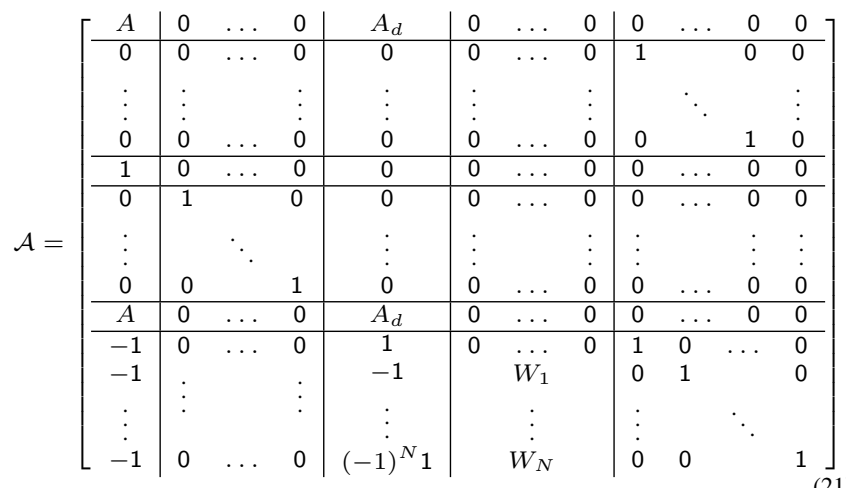


where $W_{k}=U_{k} V^{-1} E \otimes 1_{n} . V$ and $U_{k}$ are defined by equations (27) and (28) respectively.

A delay-range stability condition for time-delay systems of the form of (1) is given in the following theorem.

Theorem 4: For given positive scalars $h_{\min }$ and $h_{\max }$, if there exist positive definite matrices $P \in \mathbb{R}^{(N+1) n \times(N+1) n}$, $Q, R \in \mathbb{R}^{n \times n}$, and $n N \times n N$ matrices $S_{1}, S_{2}, S_{3}$ then the system (1) is asymptotically stable $\forall h \in\left[h_{\min }, h_{\max }\right]$ if the following LMI are satisfied:

$$
\begin{gathered}
{\left[\begin{array}{cc}
\mathcal{E} & -\mathcal{A}
\end{array}\right]^{\perp *} \Theta\left[\begin{array}{ll}
\mathcal{E} & -\mathcal{A}
\end{array}\right]^{\perp}>0, \quad S_{3}>0} \\
{\left[\begin{array}{ll}
1_{n N} & \gamma_{i} 1_{n N}
\end{array}\right]\left[\begin{array}{ll}
S_{1} & S_{2} \\
S_{2}^{*} & S_{3}
\end{array}\right]\left[\begin{array}{c}
1_{n N} \\
\gamma_{i} 1_{n N}
\end{array}\right] \leq 0, \text { for } i=\{1,2\}}
\end{gathered}
$$

where matrices $\mathcal{E}, \mathcal{A}$ and $\Theta$ are defined in (21), (24) and scalars $\gamma_{1}=\frac{1}{h_{\min }}$ and $\gamma_{2}=\frac{1}{h_{\max }}$.

Proof: We have shown that system (1) could be expressed as in Figure 1 with (19)-(20)-(21). Following the same procedure as in Theorem 3, a suitable separator is built:

$$
\Theta=\left[\begin{array}{ll}
\Theta_{1} & \Theta_{2} \\
\Theta_{2}^{*} & \Theta_{3}
\end{array}\right]
$$

with

$$
\begin{aligned}
& \Theta_{1}=\operatorname{diag}\left(0_{(N+1) n},-Q, S_{1},-h_{\text {max }}^{2} R\right), \\
& \Theta_{2}=\operatorname{diag}\left(-P, 0_{n}, S_{2}, 0_{n \times(N+1) n}\right), \\
& \Theta_{3}=\operatorname{diag}\left(0_{(N+1) n}, Q, S_{3}, R, 3 R, \ldots,(2 N+1) R\right) .
\end{aligned}
$$

The quadratic constraint related to the operator $\frac{1}{h} 1_{n}$, as well as the additional LMI (23), are based on the vertexseparator adapted from [21]. In our approach, the delay has been embedded as an uncertainty in the upper block, these latter conditions enforce $h$ to belong to the interval $\left[h_{\min }, h_{\max }\right]$. Thus, for $\nabla$ defined in (19) and the particular choice of $\Theta$ in (24), the condition (3) is satisfied. Finally, invoking Theorem 1, the aforementioned system is stable if the first condition (2) is also satisfied.

In this third theorem, we emphasize that the condition assesses the stability of (1) robustly with respect to the delay. The delay $h$ is constant but uncertain, it is assumed to belong to $\left[h_{\min }, h_{\max }\right]$. We will see through few numerical examples that the above criterion is able to detect some interval of stability (w.r.t. $h$ ) even if the delay-free system is unstable.

\section{EXAMPLES}

\section{A. First example}

Considering the following academic numerical example

$$
\dot{x}(t)=\left[\begin{array}{cc}
-2 & 0 \\
0 & -0.9
\end{array}\right] x(t)+\left[\begin{array}{cc}
-1 & 0 \\
-1 & -1
\end{array}\right] x(t-h) \text {. }
$$

This system is stable for $h_{\min }=0$, and stability is preserved up to $h_{\max }=6.1725$. Two different types of results are reported. Table I compares our pointwise stability theorem 3 with the method proposed by $\mathrm{Gu}$ et al [3] based

\begin{tabular}{|c|c|c|}
\hline Theorems & $h_{\max }$ & number of variables \\
[3] $(\tilde{N}=1)$ & 6.053 & $7.5 n^{2}+3.5 n$ \\
[3] $(\tilde{N}=2)$ & 6.165 & $10.5 n^{2}+4.5 n$ \\
[3] $(\tilde{N}=3)$ & 6.171 & $14.5 n^{2}+4.5 n$ \\
{$[3](\tilde{N}=4)$} & 6.171 & $20.5 n^{2}+5.5 n$ \\
\hline \hline Th.3 $(N=0)$ & 4.472 & $1.5 n^{2}+1.5 n$ \\
\hline Th.3 $(N=1)$ & 6.059 & $3 n^{2}+2 n$ \\
\hline Th.3 $(N=2)$ & 6.166 & $5.5 n^{2}+2.5 n$ \\
\hline Th.3 $(N=3)$ & 6.1719 & $9 n^{2}+3 n$ \\
\hline Th.3 $(N=4)$ & 6.17250 & $13.5 n^{2}+3.5 n$ \\
\hline
\end{tabular}

TABLE I

PoINTWISE METHOD: MAXIMAL ALLOWABLE DELAY $h_{\max }$ FOR SYSTEM (25).

\begin{tabular}{|c|c|c|}
\hline Theorems & $h_{\max }$ & number of variables \\
\hline \hline$[24],[11],[12]$ & 4.472 & $1.5 n^{2}+1.5 n$ \\
{$[23], D_{d}=2$} & 5.71 & $4 n^{2}+2 n$ \\
{$[23], D_{d}=3$} & 5.96 & $6.5 n^{2}+2.5 n$ \\
{$[23], D_{d}=4$} & 6.05 & $10 n^{2}+3 n$ \\
{$[13]$} & 4.97 & $69 n^{2}+5 n$ \\
{$[14]$} & 5.02 & $18 n^{2}+18 n$ \\
{$[19]$} & 5.120 & $7 n^{2}+4 n$ \\
{$[17]$} & 6.1107 & $1.5 n^{2}+9 n+9$ \\
\hline Th.4 $(N=1)$ & 6.058 & $6 n^{2}+2 n$ \\
\hline Th.4 $(N=2)$ & 6.157 & $13.5 n^{2}+4.5 n$ \\
\hline Th.4 $(N=3)$ & 6.169 & $27 n^{2}+6 n$ \\
\hline Th.4 $(N=4)$ & 6.1724 & $45.5 n^{2}+7.5 n$ \\
\hline
\end{tabular}

TABLE II

DELAY-RANGE METHOD: MAXIMAL ALLOWABLE DELAY $h_{\max }$ FOR SYSTEM (25).

on well-known discretized complete Lyapunov functional which is also a pointwise stability result. Our criterion gives similar results but with much less decision variables. Note that for $N=0$, the classical upper-bound 4.472 based on the Jensen's inequality is recovered.

Table II compares the delay range stability Theorem 4 with classical results from the literature. The partitioning approach proposed by [23] based on the discrete delay decomposition gives an upperbound which tends to the analytical value even if the numerical complexity remains important The simulations show that the best results are provided by Theorem 4 .

\section{B. Second example}

Consider now the system:

$$
\dot{x}(t)=\left[\begin{array}{cc}
0 & 1 \\
-2 & 0.1
\end{array}\right] x(t)+\left[\begin{array}{ll}
0 & 0 \\
1 & 0
\end{array}\right] x(t-h) .
$$

First let notice that the delay-free system is unstable. For this simple example, analytical method can be applied and it is shown that (26) is stable for $h \in[0.10016826,1.717]$. We aim at detecting this stability interval with Theorem 4. The results are presented in Table III.

\section{CONCLUSION}

This paper has proposed two criteria for assessing the pointwise and delay-range stability of time delay systems 
TABLE III

INTERVAL OF STABILIZING DELAYS FOR SYSTEM (26)

\begin{tabular}{lll}
\hline $\mathrm{N}$ & $h_{\min }$ & $h_{\max }$ \\
\hline 0 & - & - \\
1 & 0.1006 & 1.5404 \\
2 & 0.1003 & 1.7122 \\
3 & 0.1002 & 1.7178 \\
4 & 0.1002 & 1.7178 \\
5 & 0.10016829 & 1.7178 \\
analytical & 0.10016826 & 1.7178 \\
\hline
\end{tabular}

by using a robust analysis approach. These results are based on Legendre orthogonal polynomials and Bessel inequality. It provides a sequence of LMIs conditions which are less and less conservative, at least on examples. Future work will be devoted to the proof of the conservatism reduction and to the extension of this work to the time varying delay.

Acknowledgment: the authors thank Prof. K. Gu for his valuable comments for improving the paper and for the comparison with the discretized complete Lyapunov functional method [3].

\section{APPENDIX}

This section explains how to construct the linear relation between $\tilde{\delta}_{N}[\dot{x}(t)]$ and the other components of the vector $w(t)$. First, let introduce intermediate variables:

$$
\mu_{l}=\int_{-h}^{0}\left(\frac{\theta+h}{h}\right)^{l} e^{s \theta} d \theta, \quad 0 \leq l \leq N .
$$

Then, we have $\delta_{k}=(-1)^{k} \sum_{l=0}^{k} p_{l}^{k} \mu_{l}=V_{k} \tilde{\mu}$, with

$$
\tilde{\mu}=\left[\begin{array}{lll}
\mu_{0} & \mu_{1} & \ldots \mu_{N}
\end{array}\right]^{T} \in \mathbb{C}^{(N+1) \times 1}
$$

and

$$
V_{k}=(-1)^{k}\left[\begin{array}{lllllll}
p_{0}^{k} & p_{1}^{k} & \ldots & p_{k}^{k} & 0 & \ldots & 0
\end{array}\right] \in \mathbb{R}^{1 \times(N+1)} .
$$

Defining the matrix $V \in \mathbb{R}^{(N+1) \times(N+1)}$

$$
V=\left[\begin{array}{llll}
V_{0}^{T} & V_{1}^{T} & \ldots & V_{N}^{T}
\end{array}\right]^{T},
$$

we get $\tilde{\delta}_{N}=V \tilde{\mu}$. Because $V$ is non-singular we have

$$
\tilde{\mu}=V^{-1} \tilde{\delta}
$$

Applying $\delta_{k}$ to $\dot{x}(t)$ and integrating by parts, we obtain

$$
\begin{aligned}
\delta_{k}[\dot{x}(t)]= & (-1)^{k} \sum_{l=0}^{k} p_{l}^{k} x(t)-(-1)^{k} p_{0}^{k} x(t-h) \\
& -(-1)^{k} \sum_{l=1}^{k} p_{l}^{k} \frac{l}{h} \mu_{l-1}[x]
\end{aligned}
$$

Let remark that $(-1)^{k} \sum_{l=0}^{k} p_{l}^{k}=1$ and $p_{0}^{k}=1$. Denoting

$$
U_{k}=(-1)^{k}\left[\begin{array}{llllll}
p_{1}^{k} & 2 p_{2}^{k} & \ldots & k p_{k}^{k} & 0 & \ldots 0
\end{array}\right] \in \mathbb{R}^{1 \times(N+1)},
$$

we finally have

$$
\begin{aligned}
\delta_{k}[\dot{x}] & =\int_{-h}^{0}(-1)^{k} \sum_{l=0}^{k} p_{l}^{k}\left(\frac{\theta+h}{h}\right)^{l} \dot{x}(t+\theta) d \theta, \\
& =x(t)-(-1)^{k} x(t-h)-\left(U_{k} V^{-1} E \otimes 1_{n}\right) y(t),
\end{aligned}
$$

with $y(t)=\frac{1}{h} \tilde{\delta}_{N-1}[x]$ and $E=\left[\begin{array}{c}1_{\mathrm{N}} \\ 0_{1 \times \mathrm{N}}\end{array}\right]$.

\section{REFERENCES}

[1] W. Michiels and S. I. Niculescu, Stability and Stabilization of TimeDelay Systems, An Eigenvalue-Based Approach. Society for Industrial and Applied Mathematics (SIAM), 2007.

[2] R. Sipahi, S. Niculescu, C. Abdallah, W. Michiels, and K. Gu, "Stability and stabilization of systems with time delay," Control Systems, IEEE, vol. 31, no. 1, pp. 38 -65, feb. 2011.

[3] K. Gu, V. L. Kharitonov, and J. Chen, Stability of Time-Delay Systems. Birkhäuser Boston, 2003, control engineering.

[4] V. Kharitonov and E. Plischke, "Lyapunov matrices for time-delay systems," Systems \& Control Letters, vol. 55, no. 9, pp. 697 - 706, 2006

[5] Y. Ariba and F. Gouaisbaut, "An augmented model for robust stability analysis of time-varying delay systems," Int. J. Control, vol. 82, pp. 1616-1626, Sept. 2009.

[6] J. Kim, "Note on stability of linear systems with time-varying delay," Automatica, vol. 47, no. 9, pp. 2118-2121, 2011.

[7] E. Fridman, "New Lyapunov-Krasvoskii functionals for stability of linear retarded and neutral type systems," Systems \& Control Letters, vol. 43, pp. 309-319, 2001.

[8] K. Liu and E. Fridman, "Wirtinger's inequality and Lyapunov-based sampled-data stabilization," Automatica, vol. 48(1), p. 102108, 2012.

[9] A. Seuret and F. Gouaisbaut, "Reducing the gap of the Jensen's inequality by using the Wirtinger's inequality," submitted to Automatica, 2012.

[10] — "On the use of the Wirtinger's inequalities for time-delay systems," in Proc. of the $10^{\text {th }}$ IFAC Workshop on Time Delay Systems (IFAC TDS'12), Boston, MA, USA, 2012.

[11] Y. He, Q. G. Wang, L. Xie, and C. Lin, "Further improvement of freeweighting matrices technique for systems with time-varying delay," IEEE Trans. on Automat. Control, vol. 52, pp. 293-299, Feb. 2007.

[12] H. Shao, "New delay-dependent stability criteria for systems with interval delay," Automatica, vol. 45, no. 3, pp. 744 - 749, 2009.

[13] J.-H. Kim, "Note on stability of linear systems with time-varying delay," Automatica, vol. 47, no. 9, pp. 2118 - 2121, 2011.

[14] J. Sun, G. G.P. Liu, J. Chen, and D. Rees, "Improved delay-rangedependent stability criteria for linear systems with time-varying delays," Automatica, vol. 46, no. 2, pp. 466 - 470, 2010.

[15] S. I. Niculescu and J. Chen, "Frequency sweeping tests for asymptotic stability: a model transformation for multiple delays," in IEEE Conference on Decision and Control, Dec. 1999, pp. 4678-4683.

[16] J. Zhang, C. R. Knopse, and P. Tsiotras, "Stability of time-delay systems: Equivalence between Lyapunov and scaled small-gain conditions," IEEE Trans. on Automat. Control, vol. 46, no. 3, pp. 482-486, Mar. 2001.

[17] C.-Y. Kao and A. Rantzer, "Stability analysis of systems with uncertain time-varying delays," Automatica, vol. 43, no. 6, pp. 959 - 970, 2007.

[18] D. Peaucelle, D. Arzelier, D. Henrion, and F. Gouaisbaut, "Quadratic separation for feedback connection of an uncertain matrix and an implicit linear transformation," Automatica, vol. 43, no. 5, pp. 795804, 2007.

[19] Y. Ariba, F. Gouaisbaut, and K. Johansson, "Stability interval for timevarying delay systems," in Decision and Control (CDC), 2010 49th IEEE Conference on, dec. 2010, pp. 1017 -1022.

[20] C. Briat, "Convergence and equivalence results for the Jensen's inequality - application to time-delay and sampled-data systems," IEEE Transactions on Automatic Control, vol. 56(7), pp. 1660-1665, 2011.

[21] T. Iwasaki and S. Hara, "Well-posedness of feedback systems: insights into exact robustnessanalysis and approximate computations," IEEE Trans. on Automat. Control, vol. 43, pp. 619-630, May 1998.

[22] F. Gouaisbaut and D. Peaucelle, "Robust stability of time-delay systems with interval delays," in $46^{\text {th }}$ IEEE Conference on Decision and Control, New Orleans, USA, Dec. 2007.

[23] Q.-L. Han, "A discrete delay decomposition approach to stability of linear retarded and neutral systems," Automatica, vol. 45, no. 2, pp. $517-524,2009$.

[24] F. Gouaisbaut and D. Peaucelle, "A note on stability of time delay systems," in $5^{\text {th }}$ IFAC Symposium on Robust Control Design (ROCOND'06), Toulouse, France, July 2006. 